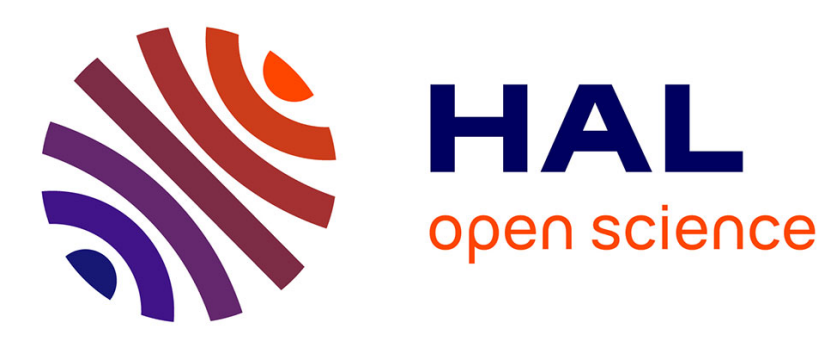

\title{
The Impact of New Mobility Modes on a City: A Generic Approach Using ABM
}

Arnaud Grignard, Luis Alonso, Patrick Taillandier, Benoit Gaudou, Tri

Nguyen-Huu, Wolfgang Gruel, Kent Larson

\section{- To cite this version:}

Arnaud Grignard, Luis Alonso, Patrick Taillandier, Benoit Gaudou, Tri Nguyen-Huu, et al.. The Impact of New Mobility Modes on a City: A Generic Approach Using ABM. 9th International Conference on Complex Systems (ICCS 2018), New England Complex Systems Institute, Cambridge, MA, Jul 2018, Cambridge, MA, United States. pp.272-280, 10.1007/978-3-319-96661-8_29 . hal-02734596

\section{HAL Id: hal-02734596 \\ https: / hal.inrae.fr/hal-02734596}

Submitted on 5 Jan 2021

HAL is a multi-disciplinary open access archive for the deposit and dissemination of scientific research documents, whether they are published or not. The documents may come from teaching and research institutions in France or abroad, or from public or private research centers.
L'archive ouverte pluridisciplinaire HAL, est destinée au dépôt et à la diffusion de documents scientifiques de niveau recherche, publiés ou non, émanant des établissements d'enseignement et de recherche français ou étrangers, des laboratoires publics ou privés. 


\title{
The Impact of New Mobility Modes on a City: A Generic Approach Using ABM
}

\author{
Arnaud Grignard ${ }^{1(\bowtie)}$, Luis Alonso ${ }^{1}$, Patrick Taillandier ${ }^{2}$, Benoit Gaudou ${ }^{3}$, \\ Tri Nguyen-Huu ${ }^{4,6}$, Wolfgang Gruel ${ }^{5}$, and Kent Larson ${ }^{1}$ \\ 1 MIT Media Lab, City Science, Cambridge, USA \\ agrignard@gmail.com \\ 2 MIAT, University of Toulouse, INRA, Toulouse, France \\ 3 University Toulouse 1 Capitole, UMR 5505 IRIT, CNRS, Toulouse, France \\ ${ }^{4}$ UMMISCO, IRD, Bondy, France \\ 5 Institute for Mobility and Digital Innovation, \\ Stuttgart Media University, Stuttgart, Germany \\ ${ }^{6}$ IXXI, ENS Lyon, Lyon, France
}

\begin{abstract}
Mobility is a key issue for city planners. Being able to evaluate the impact of its evolution is complex and involves many factors including new technologies like electric cars, autonomous vehicles and also new social habits like vehicle sharing. We need a better understanding of different scenarios to improve the quality of long-term decisions. Computer simulations can be a tool to better understand this evolution, to discuss different solutions and to communicate the implications of different decisions. In this paper, we propose a new generic model that creates an artificial micro-world which allows the modeler to create and modify new mobility scenarios in a quick and easy way. This not only helps to better understand the impact of new mobility modes on a city, but also fosters a better-informed discussion of different futures. Our model is based on the agent-based paradigm using the GAMA Platform. It takes into account different mobility modes, people profiles, congestion and traffic patterns. In this paper, we review an application of the model of the city of Cambridge.
\end{abstract}

Keywords: Agent-based modeling $\cdot$ Mobility $\cdot$ City Science

\section{Introduction}

In recent years, we have seen significant changes in the way we travel. These changes are partially driven by new technologies like electric vehicles, but also by new usage concepts such as car-sharing or ride-hailing [8]. It is anticipated that these changes will accelerate in the coming years - with wide-spread adoption of shared, autonomous and electric vehicles. For cities, it is crucial to proactively manage this development. However, this task is challenging because of the speed

(C) Springer Nature Switzerland AG 2018

A. J. Morales et al. (Eds.): ICCS 2018, SPCOM, pp. 272-280, 2018.

https://doi.org/10.1007/978-3-319-96661-8_29 
of the development and because the mobility system has many dependencies e.g., to the cities' infrastructure or its economic and social systems. Simulations can be powerful tools to test and to communicate the impact of decisions in the field of mobility. Numerous simulation tools exist to investigate current traffic questions, but few are able to examine the impact of future mobility systems. Most of the existing tools focus on specific problems and are limited to existing mobility modes.

Using meaningful simulation models might help to better understand and evaluate the effects of new services on different stakeholders in cities. Service operators might use these simulations to support the development-process of their services, cities can use them to experiment with regulatory variables like subsidies, taxes, or congestion charges, while citizens can investigate the effects on traffic-flows in their neighborhood. New mobility options are currently being developed and neither their characteristics nor the behavioral changes they create can be measured or predicted in an exact way. That is why methods to investigate the impact of these options need to deal with a high-level of uncertainty. A method to reduce uncertainty is to integrate a multitude of stakeholders into the discussion. Modeling systems are needed to quickly create, change and test different scenarios.

We propose an agent-based model (ABM) that allows the modeler to easily integrate new transportation modes. The main goal of this model is to show the impact of different mobility modes on traffic flow and congestion. We aim to address other aspects like housing or energy flows in the future. The model, implemented with the GAMA modeling platform [6], is developed as an open source project that also allows the modeler to easily develop and change mobility scenarios. We describe a framework which can be adapted easily for different contexts and we describe its application in a case study. The focus of our work is on the quick development of meaningful scenarios and the support of discussions between stakeholders rather than creating exact modeling results. The paper is structured as follows: Sect. 2 presents the context of this work. Section 3 is dedicated to the presentation of the model using the ODD protocol [7]. Section 4 presents a case study and its output. Section 5 concludes and presents perspectives.

\section{Limits of the Existing Tools for Traffic Simulation}

To investigate the complex relationships of mobility, a multitude of tools are used. Among them System Dynamics is used to investigate complex problems, to model the relationship between transportation systems [13], and to explore the impact of new systems like autonomous vehicles [9]. Questions of current and future mobility systems are also examined with the help of mathematical methods $[2,12]$. However Agent Based Modelling remains one the most popular approaches and is the base to many frameworks for traffic simulation. Among them MATSim [3] is an open-source platform with different features dedicated to traffic simulation which can be defined through the use of new modules in JAVA. 
SUMO [11] proposes many advanced features that can be developed and enriched using $\mathrm{C}++$. However, for modelers without high-level programming skills, adapting these platforms to specific application contexts, such as the integration of new transport modes, is very difficult and out of reach. As a consequence, many models are still developed from scratch (e.g. $[10,15]))$ which is time consuming and limits the re-usability of the models.

In order to face this difficulty, some authors [4,5] used a generic modeling platform to develop their models. The interest is to facilitate the comparison of models and their re-usability. One of the existing generic platforms that is particularly adapted to develop transportation models is the GAMA platform [6]. GAMA provides modelers with a complete modeling language and an integrated development environment that permits the user to build models in a rapid and easy manner. The GAMA platform provides different features that can be used by modelers to develop transportation models. In particular, it is possible to simply load GIS data, define graphs from polyline geometries, compute the shortest paths and move agents on a network. Additionally, some movement behaviors are already pre-coded and can be easily reused. Many transportation models were produced in the last years with the GAMA platform [14]. However, these models have not focused on the impact of future mobility options and they have not provided a generic framework to investigate potential impacts of these options. We use the GAMA platform to create a framework that can be adapted easily to investigate how the discussion about possible solutions is changed by models that are adaptable during that discussion. Our focus is to stimulate a well-founded discussion around different scenarios and not to model the situation as exact as possible. The discussion is supposed to generate promising scenarios that are meant to be investigated more in detail.

\section{Model Description}

Our model aims to simulate the mobility-behavior of city dwellers and create meaningful output for the discussion around new mobility services. It offers different configurations and focuses on three main categories of urban travel: non motorized transport, public transit and private motorized transport. This allows us to investigate the impact of new mobility modes and to observe behavioral changes over time considering different plans. It also reveals how urban forms and transportation infrastructure impact travel patterns.

\subsection{Entities, State Variables, and Scales}

We list here the main variables used in the model. The environmental variables are the following:

- Transport Network: A graph on which agents can travel during the simulation. Network edges have the following attributes: type: the type of infrastructure (road, rail), max speed: the maximum authorized speed on the network 
segment, capacity: the capacity in terms of agents (people, cars), proportional to the length of the network segment, current concentration: the number of people on the network segment.

- Building: each building is represented by a polygon and has three attributes: usage (Residential or Office), scale (Small (S), Medium (M), or Large (L)), category (Restaurant, Nightlife, Gathering Places, Cultural, Shopping, High School, University, Office, Residential, or Park).

- Mobility Hub: a place where people can enter/exit a mobility mode, such as a bus stop or train station. It contains one single attribute: waiting_people, the list of people waiting at this mobility hub.

The agents species used in the models are:

- People: People have several activities during the day and move around the city accordingly. People agents' behavior is to determine the location of the next activity, choose a mobility mode and move towards that location. Their attributes include: profile: the profile of the agent (e.g. High School Student, Executives, Retirees), destination: the final destination that the agent wants to reach, living_place: its living place, objectives: the schedule of objectives during the day, current_objective: its current objective, mobility_mode: the mobility mode currently used, possible_mobility_modes: the list of the mobility modes that are available to that agent.

- Transit Vehicle: Public transit vehicles follow a route and can embark/ disembark people. This species has 3 attributes: stops: the list of stops, stop_passengers: the list of people waiting at each stop, my_target: the current target of the vehicle (iteration of the list of stops).

\subsection{Process Overview and Scheduling}

The dynamics of the model is based on 3 consecutive steps: (1) management of traffic jams by the network agents, (2) behavior of the transit agents and (3) behavior of the people agents. Each step of the simulation represents $1 \mathrm{~min}$. The management of the traffic jam is based on the following rule: the more people agents move on a network segment, the lower their speed. The transit vehicle agents have a simple behavior: according to their schedules, they move from hub to hub and pick up the people agents that are waiting at each station. The behavior of the people agents is defined by their list of objectives which defines their schedule. Figure 1 gives an example: an agent wakes up, (1) goes to work and stays there for a while, (2) goes to lunch, (3) gets back to work, (4) goes to dinner and finally (5) travels back home.

Whenever an agent changes its activity, it may need to move to a new location, determine a transportation mode and compute the path to its destination. Factors like travel time or price can influence the agent's mode choice. The agents may weigh those factors differently, depending on their profile and the type of activity.

The computation of the mode of transportation is performed each time an agent decides to move. The algorithm consists of three steps: (i) Determination 
of the possible modes of transportation. This step relies on simple considerations, such as whether or not agent owns a car. (ii) Computation of factors related to each of possible mode. Currently, we consider the following factors: travel-time, price, and difficulty. Difficulty represents amount of effort that is needed: walking to a mobility hub and waiting may be seen as more difficult and time consuming than using one's own car. (iii) Choice of the mode of transportation. Each agent will determine its mode of transportation, based on its own characteristics (profile), the purpose of the trip and the indicators computed at step 2.

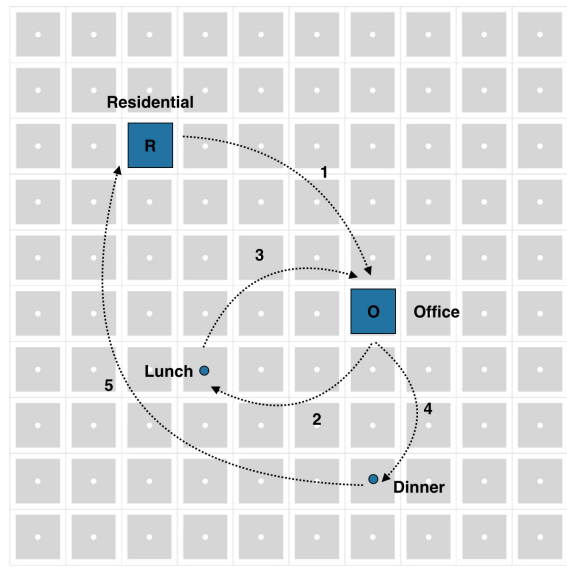

Fig. 1. Model process

Step (iii) consists in attributing a score to each transportation mode, according to a Travel Route Initialization formula based on a linear combination of the considered factor, with weights depending on the context of the travel (social category of the agent, purpose of the travel). For example, a student is more likely to use a bike than a retired person, hence the student will have a greater weight for using a bike. This Travel Route Initialization formula entirely relies on a table of weights that need to be determined for each case study. It provides the following advantages: firstly, it relies on simple mathematical considerations that are understandable by end users and stakeholders. Secondly, it provides flexibility, as it is possible to add, remove or modify the factors considered. Finally, it is easy to compute the table of weights from the data, either from individual surveys or raw mobility data. In the later case, the weight table can be determined by a multinomial logistic regression based with chosen predictors. All of this makes this approach a general framework that can easily modified and adapted to suit the requirements for different case studies.

We present here a small example with two profiles: college student and midcarrier workers. The values in Table 1a represent how important each category is. A positive (resp. negative) value means a positive (resp. negative) influence. Here the cost of travel has a very negative influence for the college student, while 
Table 1. Example for two profiles and an imaginary trip

\begin{tabular}{|l|l|l|l|}
\hline Profile & Price & Time & Difficulty \\
\hline College student & -0.9 & -0.2 & -0.65 \\
\hline Mid-carrier worker & 0 & -1 & -1 \\
\hline
\end{tabular}

(a) Weights

\begin{tabular}{|l|l|l|l|}
\hline Mode & Bike & Car & Bus \\
\hline Price & 0 & 8 & 1.5 \\
\hline Time & 40 & 10 & 20 \\
\hline Difficulty & 0.1 & 0.2 & 0.5 \\
\hline
\end{tabular}

(b) Example values

it does not matter at all for the mid-carrier worker. Table $1 \mathrm{~b}$ shows values for an imaginary trip.

From those tables, we compute a score with the above formula, shown in 2 . Each agent will chose the mode corresponding to the best score, i.e. bus for the student and car for the mid-carrier worker.

Table 2. Computed scores

\begin{tabular}{l|l|l|l}
\hline Profile & Bike & Car & Bus \\
\hline College student & -8.065 & -9.33 & -5.65 \\
\hline Mid-carrier worker & -40.1 & -10.2 & -20.5 \\
\hline
\end{tabular}

\subsection{Initialization and Input Data}

To initialize the model, the following steps have to be taken into account: (1) the building and mobility network agents are created from GIS files (shapefiles); (2) the different mobility modes are generated from a csv file that provides information about price, time traveled (speed, wait-time), and difficulty of use; (3) the people agents are created by reading two csv files: one that gives information about the agents' distribution and the probabilities to have the different mobility modes (e.g. bike or car). The second one contains information about the agents' typical schedules (activities for each hour); (4) the weights per profile and per activity are imported from a csv file; (5) the people agents are created and their location is determined based on data from the profile files.

\section{Case Study: City of Cambridge - Kendall Square}

The model described early in this paper has been applied in a larger project called CityScope Volpe developed by the City Science group at the MIT Media Lab [1] as shown in Figs. 2 and 3. MIT was recently chosen as the partner to redevelop the 14-acre site of Volpe, which formerly housed the U.S Department of Transportation. CityScope is a platform for shared, interactive computation for urban planning. It uses LEGO bricks as a tangible interface to the computational models and aims to interactively engage stakeholders in the city-planning-process. 


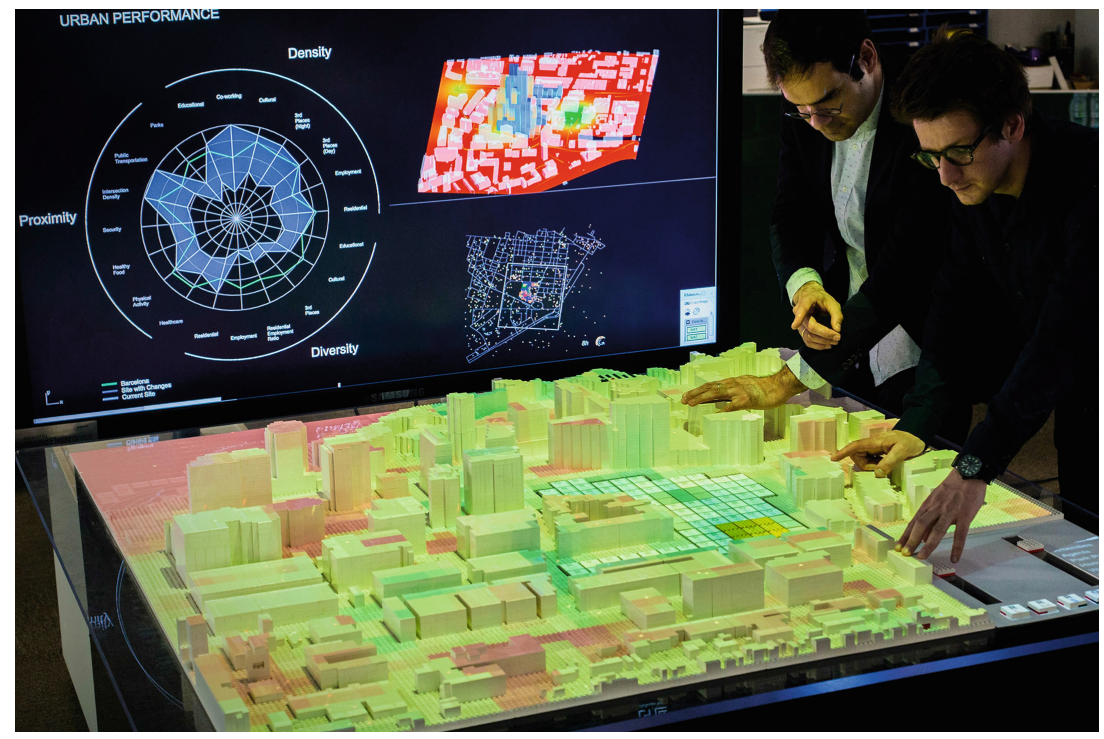

Fig. 2. CityScope: a data-driven interactive simulation tool for urban design.

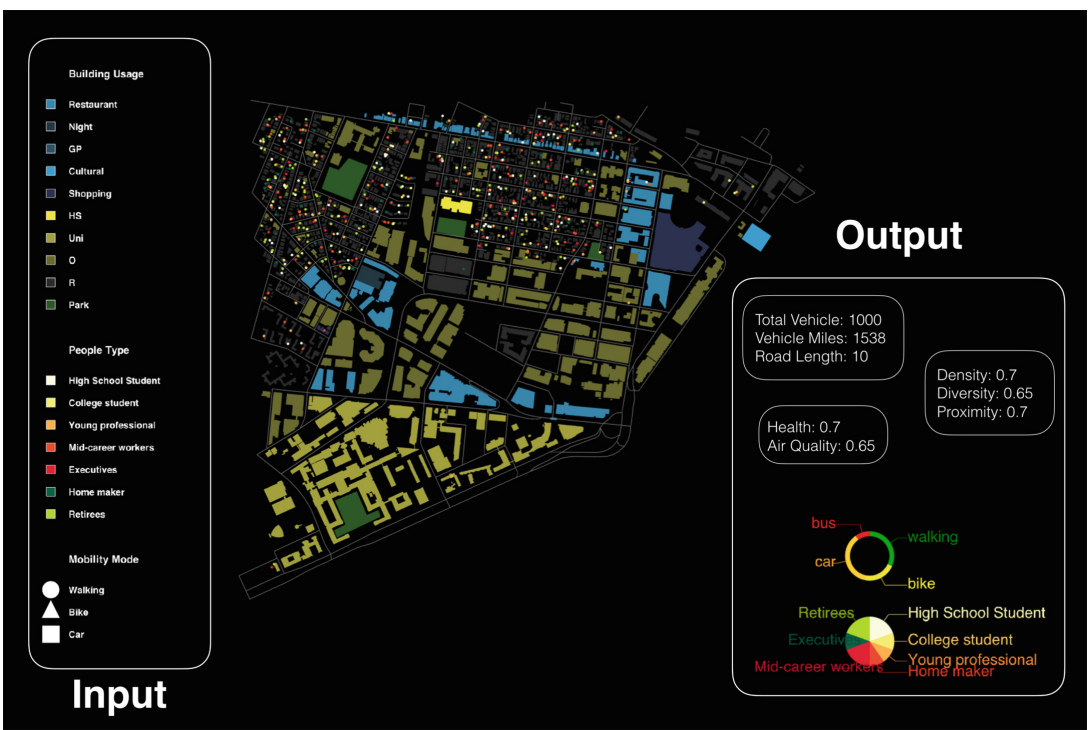

Fig. 3. Model overview.

Walkability, energy or economic activity models are used on the CityScope platform to solve spatial design and urban planning challenges. The mobility model focuses on three main aspects: total number of vehicles, vehicle miles and hours traveled, road length and congestion. The CityScope platform was used in 
several workshops in order to discuss different suggestions for the site. We applied the process that has been described before to initialize the model. For the building and mobility network (step 1), we used the proposals from the design stage of the project. For the mobility modes (step 2), we adapted information that is available from the Massachusetts Bay Transportation Authority. The people agents (step 3) could be configured by the stakeholders during the workshops. This allows for different ideas about zoning and density to be discussed. For the weights per profile, we adapted census-data for the Boston area (step 4). In our workshops, the stakeholders could change different parameters of our model by using the interactive LEGO interface. A change of the parameters resulted in a re-computation of the model.

\section{Conclusion and Discussion}

In the paper, we described a generic framework to investigate the impact of mobility modes on different aspects of daily life. It was designed to be easily adaptable to all cities and to easily integrate new mobility modes to investigate their possible impacts. Our case study showed that without programming-skills it is possible to create an ABM model that can be used to create meaningful output for the discussion around the design of mobility solutions. As our models come with many simplifications and focus on an unknown future, many uncertainties remain. However, the workshops revealed that the models contribute to a better understanding of the mobility system, even it is unclear how well specified the models need to be in order to take the optimal decision. It can be assumed that this is different for every project, and that it makes sense to refine the models during the process and to conduct in-depth investigations with different methods for the most promising alternatives. Although the creation of the model was possible without programming-skills, creating a mobility model is still not a trivial task. In order to integrate more people in the discussion about livable cities and to leverage the wisdom of crowds, the process needs to be further simplified. The stakeholders that took part in our workshops accepted our models. Despite the limitations of the model, workshop participants valued the impression of future scenarios, complex relationships became obvious and changing input parameters had direct input on the scenarios. They assume that this could lead to better decision making, than decisions that are either driven by gut-feelings or by experts who use tools that are hard to understand.

\section{References}

1. Alonso, L., Zhang, Y., Grignard, A., Noyman, A., Sakai, Y., ElKatsha, M., Doorley, R., Larson, K.: Cityscope: a data-driven interactive simulation tool for urban design. Use case volpe. In: ICCS 2018 (2018, to be Published)

2. Alonso-Mora, J., Samaranayake, S., Wallar, A., Frazzoli, E., Rus, D.: On-demand high-capacity ride-sharing via dynamic trip-vehicle assignment. Proc. Natl. Acad. Sci. 114, 462-467 (2017) 
3. Balmer, M., Rieser, M., Meister, K., Charypar, D., Lefebvre, N., Nagel, K., Axhausen, K.: MATSim-T: architecture and simulation times. In: Multi-Agent Systems for Traffic and Transportation Engineering, pp. 57-78 (2009)

4. Czura, G., Taillandier, P., Tranouez, P., Daudé, É.: Mosaiic: city-level agent-based traffic simulation adapted to emergency situations. In: Proceedings of the International Conference on Social Modeling and Simulation, plus Econophysics Colloquium 2014, pp. 265-274. Springer (2015)

5. Fosset, P., Banos, A., Beck, E., Chardonnel, S., Lang, C., Marilleau, N., Piombini, A., Leysens, T., Conesa, A., Andre-Poyaud, I., et al.: Exploring intra-urban accessibility and impacts of pollution policies with an agent-based simulation platform: GaMiroD. Systems 4(1), 5 (2016)

6. Grignard, A., Taillandier, P., Gaudou, B., Vo, D.A., Huynh, N.Q., Drogoul, A.: GAMA 1.6: advancing the art of complex agent-based modeling and simulation. In: International Conference on Principles and Practice of Multi-Agent Systems, pp. 117-131. Springer (2013)

7. Grimm, V., Berger, U., DeAngelis, D.L., Polhill, J.G., Giske, J., Railsback, S.F.: The ODD protocol: a review and first update. Ecol. Model. 221(23), 2760-2768 (2010)

8. Gruel, W., Piller, F.: New vision for personal transportation. MIT Sloan Manage. Rev. 57(2), 20-24 (2016)

9. Gruel, W., Stanford, J.M.: Assessing the long-term effects of autonomous vehicles: a speculative approach. Transp. Res. Procedia 13, 18-29 (2016). http://www.sciencedirect.com/science/article/pii/S2352146516300035, towards future innovative transport: visions, trends and methods 43rd European Transport Conference Selected Proceedings

10. Horn, M.: Multi-modal and demand-responsive passenger transport systems: a modelling framework with embedded control systems. Transp. Res. Part A Policy Pract. 36, 167-188 (2002)

11. Krajzewicz, D., Erdmann, J., Behrisch, M., Bieker, L.: Recent development and applications of SUMO - Simulation of Urban MObility. Int. J. Adv. Syst. Meas. 5(3\&4), 128-138 (2012)

12. Pavone, M., Smith, S.L., Frazzoli, E., Rus, D.: Robotic load balancing for mobilityon-demand systems. Int. J. Robot. Res. 31(7), 839-854 (2012)

13. Shepherd, S.: A review of system dynamics models applied in transportation. Transp. B Transp. Dyn. 2(2), 83-105 (2014)

14. Taillandier, P.: Traffic simulation with the gama platform. In: International Workshop on Agents in Traffic and Transportation, pp. 8-p (2014)

15. Tranouez, P., Daudé, E., Langlois, P.: A multiagent urban traffic simulation. J. Nonlinear Syst. Appl. 3(2), 98-106 (2012) 